definitive or a differential diagnosis about possible aetiology of underlying liver disease. However $98 \%$ (47/48) of patients with cancer had a diagnosis on histology. Only 53\% (9/17) patients with chronic hepatitis had severity scoring (Ishak staging/grading).

Conclusion About one third of liver biopsies did not have diagnosis or discussion about a differential diagnosis. This number goes up to $47.5 \%(28 / 59)$ if we exclude malignancies. $9 / 28$ specimens were sent to a tertiary centre and reported by a liver pathologist who gave a definite or differential diagnosis in all cases. The mortality associated with percutaneous liver biopsy ranges between 0.13 and $0.33 \%$. from an audit from UK district general hospital. With the advent of fibroscan there is less need to perform liver biopsies except in diagnosing malignancies or in hepatitis of unknown/unclear aetiology. From our study we conclude that non-cancer liver biopsies should be reported by pathologists with subspecialist interest in hepatology or the procedure should be performed in a tertiary hospital to give the clinician an accurate diagnosis to aid treatment.

Disclosure of Interest None Declared.

\section{PTU-095 LIVER LESIONS ON ULTRASONOGRAPHY: IS IT CANCER OR NOT?}

doi:10.1136/gutjnl-2013-304907.185

1."B Krishnan, 'H Alexander, 'M Abayalingam, 'A Mitra, 'V Sehgal, ' $\mathrm{K}$ Besherdas. 'Chasefarm Hospital, London, UK

Introduction The ultimate goal of the United Kingdom Cancer plan is to 'offer patients a maximum one month wait from an urgent referral for suspected cancer to the beginning of treatment'. The North London Cancer Network has devised and implemented a suspected cancer referral form for General Practitioners in London for patients to be seen within two weeks of referral to secondary care. One group referred on the suspected cancer referral form is patients with a liver lesion on ultrasound(USS). However, for a service to be effective it is essential that it is not overloaded with inappropriately referred patients.

Methods The aim of the study was to assess the appropriateness and findings in patients referred to secondary care with a liver lesion on USS. A retrospective analysis of all patients referred to the gastroenterology clinic with a liver lesion on USS on the suspected cancer referral form during a 12 month period from 1st January 2011 at a District General Hospital in North London(Chasefarm Hospital) was performed. Data was collected using medical records and electronic patient results.

Results In the study period, a total of 379 patients were referred with suspected upper GI cancer. Of these 39(10.3\%) were specifically referred in view of an abnormal liver lesion. The USS findings which prompted a referral was liver metastasis in $10(26 \%)$, hypoechoic lesion in $7(18 \%)$, liver mass in $6(15 \%)$, hemangioma in $4(10 \%)$, area of increased echogenicity in $3(8 \%)$, isoechoic lesion in $3(8 \%)$, cyst in $2(5 \%)$ and hyperechoic lesion, echo poor area, echo bright area, calcified foci in 1 patient each(3\%). All patients were seen in the clinic within 2 weeks and $29(74 \%)$ had a CT and 7(18\%) had an MRI scan.

After review of images and/or histology in the clinic/multidisciplinary meeting, 10 patients had normal scan, 9 patients had hemangioma, 8 had liver metastasis, 5 had liver cysts, 2 patients did not attend follow up, 1 patient was too frail for further imaging, 1 patient each had hepatoma, adenoma, focal nodular hyperplasia and focal fat sparing.

$7 / 10(70 \%)$ patients with suspected metastasis on USS had confirmed metastatic disease on subsequent imaging. The 2 patients with liver cysts on USS had cysts on CT scan.

Conclusion The majority (77\%) of patients with suspected cancer referred to the service did not have cancer. However, $70 \%$ of patients with suspected liver metastasis on USS had this confirmed on CT scan. Simple cysts on USS proved to be cysts on CT. We recommend that if initial USS suggests metastases it is likely to be so and would recommend CT staging and tumour markers prior to outpatient review to expedite management. USS is highly sensitive in differentiating a cyst from a solid lesion and patients with cysts on USS can be offered a routine appointment rather than being seen as a potential cancer referral.

Disclosure of Interest None Declared

\section{PTU-096 METABOLIC BONE DISEASE IN MILD CHRONIC LIVER DISEASE- IS IT WORTH CHECKING?}

doi:10.1136/gutjnl-2013-304907.186

1."B Patel, ${ }^{2} \mathrm{~S}$ McLaughlin, ${ }^{3} \mathrm{E}$ Williams, ${ }^{3} \mathrm{~S}$ Al-Shamma. 'Medicine; ${ }^{2}$ Gastroenterology; ${ }^{3}$ Hepatology, The Royal Bournemouth Hospital, Bournemouth, UK

Introduction Liver cirrhosis (LC) is increasing in frequency and can cause significant morbidity including metabolic bone disease (MBD) namely osteopaenia and osteoporosis, risk factors of fractures. It is vital that patients with $\mathrm{MBD}$ are identified and commenced on appropriate treatment. Whilst studies have investigated the prevalence of $\mathrm{MBD}$ in cirrhotics awaiting transplantation, the exact frequency of MBD in mild LC is not known. We aimed to assess the frequency of MBD in patients with mild LC (MELD score $<16$ ) who had undergone DEXA scanning at our instituation to ascertain whether routine screening is worthwhile.

Methods This was a single-centre retrospective study. From a radiology database, we identified 62 patients with histologically, radiologically and/or clinically diagnosed LC who had undergone a DEXA scan between May 2007 \& June 2012. Osteopaenia and osteoporosis were defined using WHO criteria. Contemporaneous bloods were used to calculate the MELD. A similar number of age and sexmatched patients with histologically-confirmed coeliac disease (CD) who had had a DEXA scan were identified to act as a comparison group. The same scanner was used for all patients.

\section{Results Patient characteristics}

We identified 61 patients with LC and 45 with CD. The most common aetiologies were ALD (52\%) and PBC (23\%). The mean age for cirrhotics was 60.1 years (range 33-78) with $64 \% \mathrm{M}$ and $36 \% \mathrm{~F}$. The mean MELD was $10.7 \pm 4.1$. For those with $C D$ the mean age was 52 years (range 17-82) with $27 \% \mathrm{M} \& 73 \% \mathrm{~F}$.

\section{Patients with osteoporosis \& osteopenia}

Of the cirrhotcis, $57 \%$ had MBD (36\% osteopenia, $21 \%$ osteoporosis) compared to $62 \%$ of patients with CD (42\% and $20 \%$ respectively). $57 \%$ of patients with ALD had MBD compared to $71 \%$ of patients with PBC. The mean age of those with LC and osteoporosis was 62.4 years (range $47-78$ ) with $54 \%$ of patients being male. For those with $\mathrm{CD}$ and osteoporosis the mean age was 69 years (range 46-82) with 89\% being female.

\section{MBD in male ALD patients}

Male ALD patients were at particular risk. There was a statistically significantly higher risk of MBD in male ALD patients $(77.8 \%$; $55.8-99.8 \%)$ compared to male CD patients $(32.1 \%$; $13.1-51.2 \%$; $\mathrm{P}<0.05$.) On the other hand, female CD patients had a satistically greater risk of $\mathrm{MBD}$ vs females with ALD.

\section{MELD score correlation with T-score}

We found no correlation between the MELD score and the lumbar or hip T-score $(p=0.803$.) Therefore, DEXA screening should be offered to all LC patients regardless of disease severity.

Conclusion All patients with LC, irrespective of MELD, should undergo DEXA scanning due to the high prevalence of MBD. Male ALD patients warrant particular attention due to the particularly increased risk of $\mathrm{MBD}$ and fractures.

Disclosure of Interest None Declared 